\title{
THERMAL BEHAVIOR OF CERAMIC PARTICLES IN A GASEOUS MEDIUM AT HIGH TEMPERATURE
}

\author{
Abderrahmane AISSA ${ }^{1, *}$, Abdel-Nour ZAIM ${ }^{1}$, M SAHNOUN $^{1}$, FARES Redouane $^{3}$, M ABDELOUHAB $^{3}$, Mohammed EL \\ GANAOUI $^{2}$ \\ ${ }^{1}$ Laboratoire de Physique Quantique de la Matière et Modélisation Mathématique (LPQ3M), Université Mustapha Stambouli de Mascara, \\ Algeria \\ ${ }^{2}$ Lorraine Universities, Energetic Laboratory of Longwy (FJV/LERMAB), Henri Poincaré Institute of Longwy, France \\ ${ }^{3}$ Laboratoire de Modélisation \& Optimisation des Systèmes Industriels, Université des Sciences et de la Technologie - Mohammed \\ Boudiaf - BP 1505 - Oran - Algérie
}

\begin{abstract}
Numerical simulation of the interaction between the spherical particle and plasma gas is carried out. The aim of this study is to investigate thermal transfer between the plasma gas and solid particle during the plasma spray process and to validate the well-known empirical correlation of the Ranz and Marshall. In the conditions of molten or semi-molten states of prepared substrate, the medium (plasma jet) can affect the high velocities of particles. On the basis of direct numerical simulation, the computational analysis has been carried out by using computational fluid dynamics (CFD) of heat transfer in atmospheric pressure and midtemperature range $(3000 \mathrm{k}-12000 \mathrm{k})$ of a plasma flow over a spherical particle. Our proposed model improves correlation with experiments compared to the existing approaches in the literature.
\end{abstract}

\section{INTRODUCTION}

Thermal spraying is a process to treat the material surface in a dry environment to amend the properties of the support material. The thermal protection properties such as the wear resistances and friction necessary to protect against corrosion and biocompatibility, are Improved by these treatments. Thermal spraying uses heat exchanges carrier gas which transports and accelerates fine particles about 5 to 100 micrometers of molten material by a high temperature of plasma gas on a surface coat. The droplets are deposited on the substrate and then solidified. The particles accumulation on the substrate creates a coating. The connections between the substrate and the deposited layer are entirely mechanical. The material to be deposited may be in the form of powder, wire or rod. The energy source may be provided with flame or electric arc plasma jet. Fiszdon [5] established a model in finite differences to study heat transfer to the alumina particles injected into a plasma jet of the argon-hydrogen. It takes into account the internal heat conduction and the phase changes in a particle. Chen and Parker [6] have studied heat transfer characteristics in the case of the water droplets and particles of alumina, graphite and tungsten injected into argon, nitrogen or argon-hydrogen plasma jets. The model takes into account the internal particle heat conduction and convective effects to their surfaces. Varacalle et al. modelled [6] the treatment particles of nickel, aluminum and chrome oxide in argon-helium plasma jet under open-air conditions. The model considers the gas plasma as single-zone k- $\varepsilon$ turbulence. In the Process algorithm, the authors adopted the same corrections of the Lee et al [7]. in terms of heat transfer coefficient. Nam [7] studied the forces acting on a particle in a stationary argon plasma jet where the turbulence is modelled by a k- $\varepsilon$ model. It introduced the Basset force, added mass, and rotation, of Saffman, Rubinov and Keller to calculate trajectories of particles of aluminum, nickel and tungsten. The drag coefficient is not corrected. Only the Nusselt number is corrected according to Lewis and Gauvin [6]. The author compared the results of Lagrangian and Eulerian approaches and found that they give a close temperatures and speeds. In case the thermal conductivity of the material is low, or the residence time is long and the Reynolds number is low, they induced a temperature gradient in the particle. Zhuang and al. [8] have estimated the dimensions of the task of zirconium particles impact on the substrate projected by hydrogen-nitrogen plasma jet under openair conditions. The model is based on the resolution of the corresponding continuity, momentum, energy and mass conservation equations of the gas plasma, and 
plasma-particle. The charge effect of the particles is taken into account. Comparison of the numerical results with experimental measurements by stroboscopy laser shows an agreement estimated at $25 \%$ when the drag coefficient is not corrected, this agreement reached $40 \%$ when the drag coefficient is corrected to account for the change in the properties of the gas plasma around the particle.

Laura [9] has dealt with the heat and mass transfer, in a bilayer composite particle (heart metal and ceramic envelope) in flight, projected by a direct current arc plasma jet. An Enthalpy model was used with a discretization of the equations by means of finite volumes of the second order in time and in space. The aim of this work is to determine the heat exchange between the plasma gas (Helium, Argon) and the

\section{ANALYSIS AND MODELLING}

Heat flow is most often simulated [14] via a heat transfer coefficient, $\mathrm{h}$

$$
q=h\left(T_{\infty}-T_{S}\right)
$$

Where $\mathrm{q}$ is the heat flux $\left(\mathrm{W} \mathrm{m}^{-2}\right), \mathrm{T} \infty$ is the temperature of the surrounding fluid and TS is the surface temperature of the particle. The value of $h$ depends on the geometry of the particle, the relative velocity between the particle and the fluid and transport properties of the fluid [14]. Frequently, the heat transfer coefficient is represented in non-dimensional form, using the Nusselt number, $\mathrm{Nu}$.

For the momentum transfer, the drag coefficient $C_{D}$ is expressed as a function of the Reynolds number Re of the particle, but different expressions exist according to the values of $\operatorname{Re}$ [Table1]. The corrections due to temperature gradients are not negligible and the particle trajectory can be modified.

Ranz and Marshall performed experimental measurements on free-falling Drops and showed that $\mathrm{Nu}$ can be estimated from the values of Pr and Re.

$$
N u=h \cdot \frac{d_{p}}{\bar{k}}=a+c \operatorname{Re}^{m} \operatorname{Pr}^{n}
$$

This equation has to be modified to account for the temperature dependence of the gas properties within the thermal boundary layer, for non-continuum effects and for evaporation. Hence, correction factors accounting for these effects have been introduced. Table1 summarizes some Nusselt number correlations. This is why we need to obtain a unified formulation valid for all plasma gas. In this context, we proposed a new Nusselt correlation number given us:

$$
N u=h \cdot \frac{d_{p}}{\bar{k}}=a+c \operatorname{Re}^{m} \operatorname{Pr}^{n} Y^{i}
$$

spherical particle. Indeed, in plasma sprays, metal or ceramic materials (50 microns) in a molten or semi molten state are projected at high velocity on beforehand prepared substrates. The gas reaches high temperatures ranging from $6000 \mathrm{~K}$ to $12000 \mathrm{~K}$ and ensuring particle fusion for most refractory materials. The impact velocity of the drops is relatively higher. It is difficult to describe the behavior during the impact on the substrate which is directly related to the thermal and dynamic particle histories in the flame. This dynamic and thermal behavior is described by numerical simulations to independently evaluate the axisymmetric jet flow and the particle behavior injected into it [6-12]. This study is conducted to demonstrate the effects of various pertinent parameters on the fluid flow and heat transfer characteristics.

The term $\mathrm{Y}$ introduced in equation (3) given by the formula $Y=\left(\frac{\rho_{\infty} \mu_{\infty}}{\rho_{w} \mu_{w}}\right)$ also appears in other Correlations.

In this study, the momentum and energy equations, together with the above appropriate boundary conditions (fig.1), were solved in a segregated manner using COMSOL Multiphysics software. The Values of the global characteristic quantities such as the Nusselt numbers on the particle surface were obtained through COMSOL post processing. From the obtained values $\mathrm{Nu}$ was computed and compared with the correlations with the correlations proposed by Ranz \& Marshall, Lewis \& Gauvin, Fiszdon, Lee \& Pfender, and Kalganova Table 1.

Table 1: Empirical correlations used for calculating the Nusselt's number

\begin{tabular}{|l|l|}
\hline Ranz \& Marshall [8] & $N u=2+0.6 \operatorname{Re}^{0.5} \operatorname{Pr}^{0.33}$ \\
\hline Lewis \& Gauvin [1] & $N u=\left(2+0.515 \operatorname{Re}^{0.5}\right)\left(\frac{\rho_{\infty} \mu_{\infty}}{\rho_{w} \mu_{w}}\right)^{-0.15}$ \\
\hline Fiszdon [9,11] & $N u=\left(2+0.6 \operatorname{Re}^{0.5} \operatorname{Pr}^{0.33}\right)\left(\frac{\rho_{\infty} \mu_{\infty}}{\rho_{w} \mu_{w}}\right)^{0.6}$ \\
\hline Lee \& Pfender [11] & $N u=\left(2+0.6 \operatorname{Re}^{0.5} \operatorname{Pr}^{0.33}\right)\left(\frac{\rho_{\infty} \mu_{\infty}}{\rho_{w} \mu_{w}}\right)^{0.6}\left(\frac{c_{p \infty}}{c_{p w}}\right)^{0.38}$ \\
\hline Kalganova [12] & $N u=2 \frac{\kappa_{w}}{\kappa_{\infty}}+0.5 \operatorname{Re}^{0.5} \operatorname{Pr}^{0.4}\left(\frac{\rho_{\infty} \mu_{\infty}}{\rho_{w} \mu_{w}}\right)^{0.2}$ \\
\hline
\end{tabular}

The fluid flow is modelled by using the Navier-Stokes equations for incompressible flows: 


$$
\begin{aligned}
& \nabla u=0 \\
& \rho \frac{\partial u}{\partial t}+\rho(u \nabla) u=-\nabla p+\nabla u\left[\nabla u+(\nabla u)^{T}\right]+\rho g+F
\end{aligned}
$$

Where $u$ is the velocity, $p$ the pressure, $\rho$ the density, $\mu$ is the kinematic viscosity, $g$ the gravity.

For a particle in a plasma jet, two characteristics are studied: motion (trajectory, velocity, acceleration) and thermal evolution (temperature, physical state, heat flux). When a particle and plasma are in relative motion, a drag force is given by the fluid to the particle. This force comes from current lines dissymmetry between particle upstream and downstream. This force is given by [8]

$$
F_{D}=-\frac{1}{2} C_{D} \cdot \pi \cdot \frac{d_{p}^{2}}{4} \rho_{\infty}\left|u_{\infty}-u_{p}\right| \cdot\left(u_{\infty}-u_{p}\right)
$$

The convective and conductive heat transfer is described by eq. (4):

$$
\rho C_{p} \frac{d T}{d t}+\rho C_{p} u \nabla T=\nabla(k \nabla T)+q
$$

(u) is the velocity field predicted by the model incompressible Navier-Stokes. For transport by conduction and convection, the thermal flux vector is given by:

$$
q=-K \nabla T+\nabla c_{P} T . V
$$

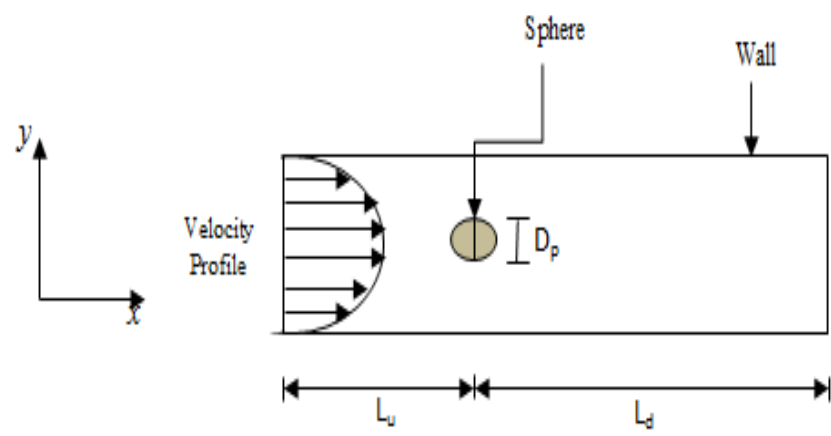

Fig.1. Boundary conditions of the study domains

The dimensionless boundary conditions are given by:

$$
\begin{array}{lll}
r=r_{s} & T=T_{s} & V=0 \\
r \rightarrow \infty & T=T_{\infty} & u=V_{\infty}
\end{array}
$$

Where $\mathrm{r}$ is the radial coordinate $r_{s}$ is the particle radius, $\mathrm{T}$ is its temperature, $\infty$ Represents the plasma gas.

\section{RESULTS AND DISCUSSION}

The present study focuses on investigating the effect of various parameters in heat transfer between spherical particle and its surrounding atmosphere at high temperature for different flow velocity.

Fig. 2 shows the contours of the streamlines and isotherm-lines for case of inlet gas velocity at $x-y$-plane of $\mathrm{z}=0$. Similar profiles of plasma velocity and temperature are found in 2D axisymmetric simulations. The balance between conduction and convection heat transfers from the hot gas to the particle, are generally neglected and particle cooling due to radiative heat losses to the surroundings governs the particle temperature. The heat transfer coefficient, $h$, between plasma and particle can be expressed in terms of the Nusselt number $\mathrm{Nu}$, linked to the numbers of Reynolds, Re, and Prandlt, Pr. Authors [table1] have proposed different expressions of $\mathrm{Nu}$ and thus of $h$. Differences are particularly important when considering monatomic gases such as Ar and $\mathrm{He}$. This is illustrated in Fig. 3 representing the calculated average Nusselt number versus plasma velocity for a alumina $50 \mu \mathrm{m}$ particle, at $\mathrm{T} \mathrm{p}=3000 \mathrm{~K}$.
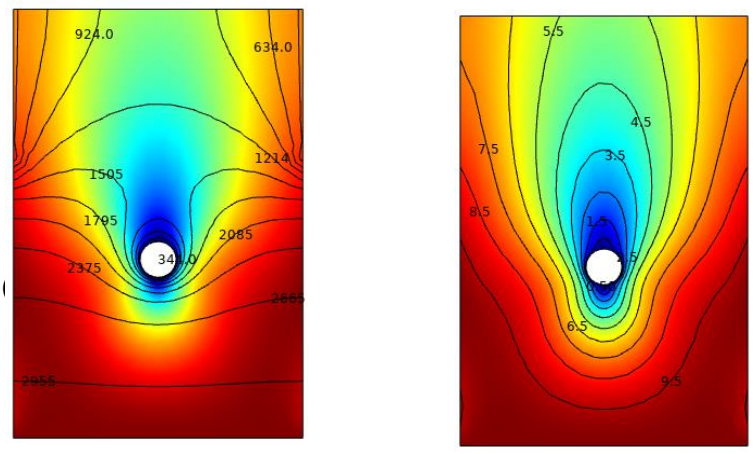

Fig.2. Illustration of the simulated temperature(left) and velocity fields(right) for $\operatorname{Pr}=0.667$.

In Fig. 3, the diagram of the average Nusselt on the particle surface has been plotted at various plasma velocity values for different gas. It can be seen that with increasing inlet velocity values, the average Nusselt number on the wall increase. For the plasma gas Helium, the average Nusselt numbers are plotted for both cases (numerical simulation and correlations proposed by the authors listed in table 1). When analyzing the numerical results obtained it can be noticed that all correlations and simulated values are lower than those obtained by the Ranz \& Marshall model.

For the argon, another mono atomic plasma gas, fig .4 shows that all correlations agree well with our results in a wide range except the cases of Lewis and Gauvin (1973) and Kalganova (1976) models. As we can notice, every 
correlation leads to different results according to the nature of the plasma gas used.
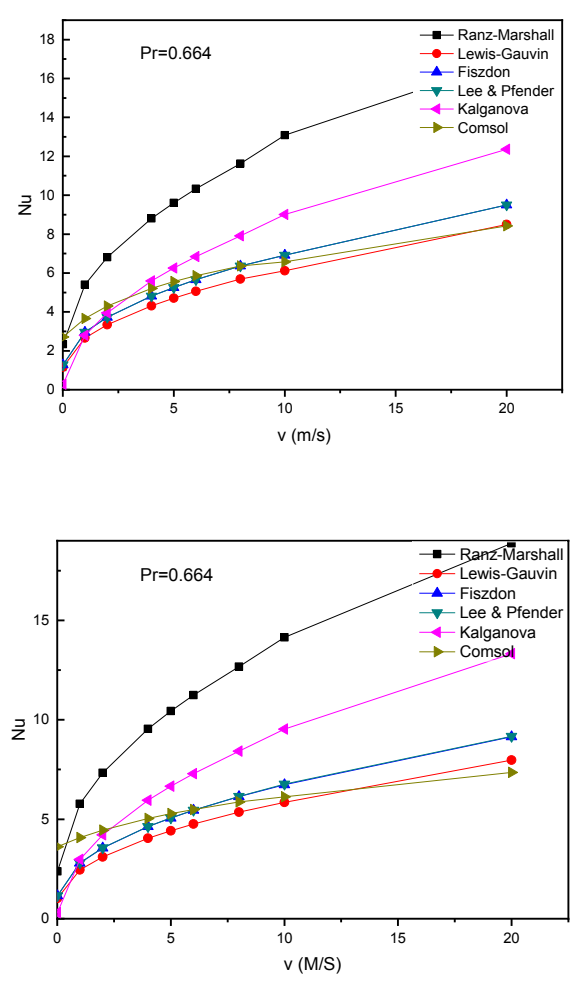

Fig.3. Comparison of the values of Nusselt number given in literature and with the values obtained from the proposed correlation for Helium
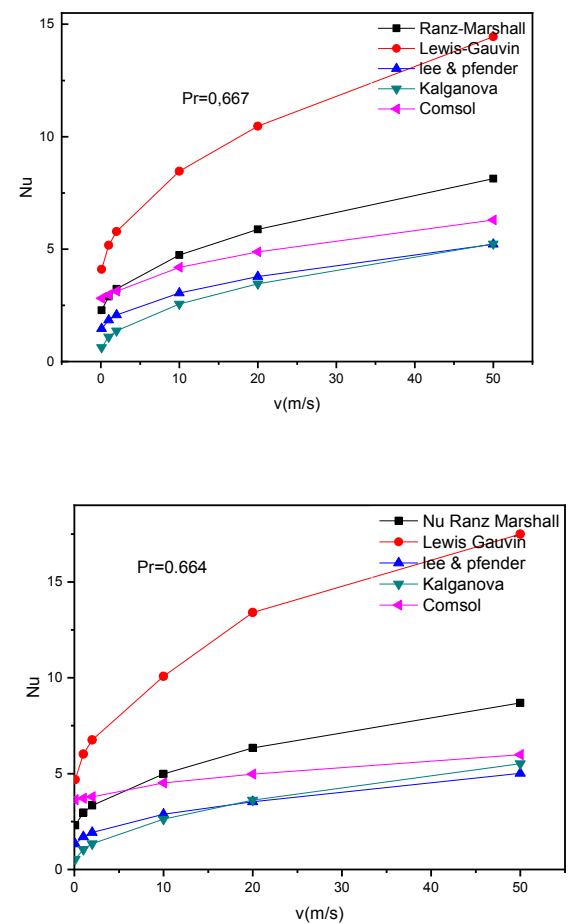

Fig.3.Comparison of the values of Nusselt number given in literature and with the values obtained from the proposed correlation for argon

\section{CONCLUSIONS}

In this study, we investigate numerically the heat transfer between a spherical particle and surrounding plasma gas at high temperature. We show that the model is validated after comparison between the present results and those of the literature. The model equations were solved using Comsol Multiphysics; a solver for partial differential Navier-Stokes equations based on a two-dimensional Finite Element Method. Following this study, it appears that the interaction of spherical particle and argon and Helium plasma jet involves several complex mechanisms. Semi-empirical correlations for heat transfer between a spherical particle and plasma jet have been analyzed. Finally, the present results are in excellent agreement with the reported published results.

\section{REFERENCES}

[1] C. Moreau, J. F Bisson, R. S. Lima, B. R Marple, Diagnostic for advanced materials processing by plasma spraying, Pure Appl. Chem., Vol.77 (2), p 443-462, (2005)

[2] A.A. Syed, A. Denoirjean, P. Denoirjean, J.C Labbe, P. Fauchais, Investigation of phenomena influencing properties of plasma sprayed ceramic-metal composite deposits, High Temperature Material Processes, 8 (2), p 253$272,(2004)$

[3] Oukach, S., and al: Numerical Study of the Spreading and Solidification of A Molten Particle Impacting Onto A Rigid Substrate under Plasma Spraying Conditions, Thermal Science Vol. 19, No. 1, pp. 277-284, (2015)

[4] Ridha Djebali, Bernard Pateyron, Mohamed El Ganaoui, A lattice Boltzmann based investigation of powder in-flight characteristics during APS process, part II: Effects of parameter dispersions at powder injection, Surface \& Coatings Technology, Vol. 220, 157-163, (2013)

[5] J. K. Fiszdon, "Melting of Powder Grains in a Plasma Flame, Int. J. Heat Mass Transfer 22, 749 (1979).

[6] Young, R. M., Pfender, E., Nusselt Number Correlations for Heat Transfer to Small Spheres in Thermal Plasma Flows, Plasma Chemistry and Plasma Processing, 7 2, pp. 211-227, (1987)

[7] S. W. Nam, Numerical analysis of heat transfer and fluid flow of a high temperature jet 
including particles in plasma spraying, Heat Transfer - Japanese Research, 22 (5), p 493505, (1993)

[8] W. H. Zhuang, D. Gray, K. Etemadi, D. M. Benenson, Study of the deposition offset in plasma spray of zirconia, Plasma Chemistry and Plasma Processing, 16 (1), p 127S-139S, (1996)

[9] M. Bouneder, Modèle des Transferts de Chaleur et de Masse dans les Poudres Composites Métal/Céramique en Projection Thermique, Thèse de l'université de Limoges, 2006
[10] Aissa, A., et al, Ranz and Marshall correlations limits on heat flow between a sphere and its surrounding gas at high temperature, Thermal science, Vol. 19, No. 5, pp. 1521-1528, ( 2015)

[11] Ranz, W. E., Marshall, W. R., Evaporation from Drops, Chem. Eng. Prog, 48 pp. 141-146, (1952)

[12] Lewis, A., Gauvin, W. H., Motion of Particles Entrained in a Plasma Jet, AICHE Journal, 19, 5, pp. 982-990, (1973) 